\title{
Retrospective Study of the Effect of Statins on the Outcome of Lung Cancer Patients, University of Cincinnati Experience
}

\author{
Ola Gaber ${ }^{1 *}$, Ihab Eldessouki ${ }^{1}$, Rammey Hassan ${ }^{1}$, Mohamed Magdy², John C \\ Morris $^{1}$, Nagla Abdel Karim ${ }^{1}$
}

\begin{abstract}
Objectives: Numerous studies addressed the effect of statin on cancer patients. The aim of this study is to define the effect of statin administration with chemotherapy on the patients' outcomes. Methods: We retrospectively researched the database of the University of Cincinnati cancer to identify lung cancer patients who received statins $(\mathrm{S}+, \mathrm{n}=41)$ during their treatment in our institute. We also, retrieved data for contemporaneously treated patients who did not receive statins ( $\mathrm{S}-, \mathrm{n}=159)$ as a control arm. Clinico-demographical data and overall survival (OS) were analyzed using Pearson's Chi-square $\left(\chi^{2}\right)$ test and Kaplan-Meier survival curves with Log-rank test. Adjustment using Cox proportional hazard ratios (HR) were done based on (age, gender, race and stage) to identify effect of statins on their outcomes. Results: The median age for S+ was 64y (IQR; 55-69) and 71.2\% of the patients were white. Histopathology was $55.4 \%$ and $31.7 \%$ for adenocarcinoma and squamous cell carcinoma, respectively. Fifty-six percent were stage IV in each study arm and the median OS was $14.9 \mathrm{~m}$. Median OS was insignificantly lower in S-ve arm (13.7 vs 15.6 months; $\mathrm{P}=0.652, \mathrm{HR}=0.91,95 \% \mathrm{CI} 0.52-1.57$ ). Our results show that after different types of adjustments, $\mathrm{S}+$ did not show survival advantage $(\mathrm{P}>0.05)$ compared to the control arm. Conclusion: While showing an increase in overall survival in patients with advanced lung cancer, the results of this study did not reach statistical significance. This could be due for the small sample size of this retrospective study.
\end{abstract}

Keywords: Lung cancer- statins- cholesterol- apoptosis- lipid metabolism- survival

Asian Pac J Cancer Prev, 20 (8), 2391-2396

\section{Introduction}

The study of occlusive vascular diseases have long established the role of Lipid metabolism in disease development (Hajar, 1995; ENDO, 2010). In the 1960s, the Framingham Heart Study provided the first widely accepted evidence that individuals with higher baseline blood cholesterol levels were more likely to experience myocardial infarction in subsequent years with blood pressure and that smoking also increased risk (ENDO, 2010). Since then, scientific research identified 3-hydroxy-3-methyl-glutaryl-CoA reductase enzyme, known as $\mathrm{HMG}-\mathrm{CoA}$ reductase, as the rate-limiting enzyme in the cholesterol biosynthesis and became the major druggable target in coronary vascular syndromes treatment (Hajar, 1995).

From a physiological stand point, cells modulate their anabolic and catabolic pathways according to nutrient availability, however, malignant cells resist physiological regulatory mechanisms of metabolism to counter stresses such as nutrient deficit. When it comes to lipid metabolism, instead of relying on the uptake of exogenous fatty acids, cancer cells increase de novo lipogenesis, thus, upregulating their signaling molecules. Several studies have identified enzymes that has significance in the role of lipid metabolism in lung cancer cells such as fatty acid synthase (FAS), stearoyl CoA desaturase 1 (SCD 1), the ABC family of transporters, and ATP citrate lyase (ACLY) (Visca et al., 2004; Cerne et al., 2010; Noto et al., 2013; Merino Salvador et al., 2017). The ratio of saturated to monounsaturated fatty acids affects cell function and membrane fluidity and studies have proposed that increased monounsaturated fatty acids can be a predictive marker for poorer prognosis(Agustsson et al., 2007), and so, SCD1 expression has been linked to malignant transformation, proliferation and survival of tumor cells (Merino Salvador et al., 2017).

Among the ABC family of transporters is the ABCA1 protein (ATP-binding cassette transporter or cholesterol efflux regulatory protein - CERP), which mediates the efflux of cholesterol and phospholipids and their transformation into apolipoprotein A1 part of high-density

${ }^{1}$ Department of Hematology-Oncology, Division of Internal Medicine, Vontz Center for Molecular Studies, University of Cincinnati, 3125 Eden Ave, Cincinnati OH 45267, United States, ${ }^{2}$ Children Cancer Hospital, Cairo, Egypt. *For Correspondence: drs_olagaber@yahoo.com 
lipoprotein (HDL). Members of the ABC family of transporters also contribute to the efflux of drugs, which could influence their intracellular concentrations and subsequently resistance to therapy (Merino Salvador et al., 2017). This was particularly for platinum-based regimens in lung cancer patients (Yoh et al., 2004). Another study looked at the distribution and accumulation of the EGFR inhibitor gefitinib in NSCLC cell lines with differing levels of ABCG2 plasma membrane levels and suggesting that the silencing or overexpression of ABCG2 affects intracellular gefitinib content by modulating its uptake (Galetti et al., 2015).

ACLY positive expression was linked to poor survivals in lung cancer (Osugi et al., 2015). However, Hanai et al., (2012) were able to show ACLY knockdown promotes apoptosis and differentiation which leads to the inhibition of tumor growth in vivo. Statins, which act downstream of ACLY in the cholesterol synthesis pathway, dramatically enhanced the anti-tumor effects of ACLY inhibition, leading to tumor regression. Thus, statin treatment affect both the PI3K/AKT and the MAPK pathways and combining it with ACLY inhibition reduced EGF receptor resistant tumor cells growth.

Consequently, Chan et al., (2003) proposed mechanisms for statins' effects on apoptosis, proliferation, invasion, and radio-sensitization of cancer cells and supported their applications as molecular targeted agents. Their antiproliferative effect was achieved through blocking G1 to $\mathrm{S}$ transition of cell cycle, and since cells located in late G1 and G2-M phases of the cell cycle are more sensitive to ionizing radiation-induced cell death, statins can potentially sensitize cells to radiation. Their apoptotic effects was through depletion of geranylgeranylated proteins (Chan et al., 2003; Graaf et al., 2004), decreased expression of the anti-apoptotic Bcl-2 and increased expression of the proapoptotic Bax. The anti-invasion properties were mediated through diminishing EGF-induced translocation of RhoA from the cytosol to the plasma membrane fraction and the actin stress fiber assembly without inhibiting the tyrosine phosphorylation of EGF receptor or c-erbB-s (Chan et al., 2003). Other studies have shown similar mechanisms (Page et al., 1993; Oudat et al., 2002; Khanzada et al., 2006; Hoque et al., 2008).

For the past 35 years the number one and number two causes of death in all persons in the United States of America have remained diseases of the heart and malignant neoplasms, respectively (C. for Disease Control, 2017). Lung Cancer, in particular has been the leading cause of death in cancer patients in the United states (Anon, n.d.; Jemal et al., 2010; Rahouma et al., 2018). The disease can have multiple subcategories and histiologies that do not seem to comply with guidelines (Karim et al., 2017, 2018; N. et al., 2017; Eldessouki et al., 2018; Eldessouki et al., 2018a; Eldessouki et al., 2018b; Gaber et al., 2019). There is a push to find novel uses for existing FDA approved drugs in the treatment of other diseases with the benefit not only being for known safety, dosage, and toxicity; but also saving time and money with regards to development (Junior, 2015; Hassan et al., 2017; N et al., 2017; Abdel Karim et al., 2018, Abdel Karim et al., 2019). Also, the advances in the biomarkers field of cancer suggest strong association between cell metabolic activity and tumor load(Gaber et al., 2018). Hereby, we introduce.

\section{Materials and Methods}

\section{Patients Data}

In a single academic-based institution (University of Cincinnati Medical Center), we retrieved our electronic medical database from January 2011 till June 2017. Patients with lung cancer, including non-small cell lung cancers (NSCLC), small cell lung cancers (SCLC) and rare histopathologic types, were retrospectively identified. Patients without a tissue diagnosis were excluded from analysis. EPIC (Epic Systems Software, Verona, WI) was implemented to collect data on patient demographics, histopathological diagnosis, clinical stage (see Table 1), duration of patients taking medication, date of death and other variables were collected. $\mathrm{S}+$ patients were identified and $\mathrm{S}-$ were used as the control group. Death was the end point of the study.

\section{Survival and follow-up data}

OS is the time from date of diagnosis to the date of death from cancer or other causes or date of last follow-up28,30. Median follow-up was 17 months (95\% confidence intervals $(95 \% \mathrm{CI})=11.14-24.26)$ for the whole group, while it was 25.63 months (95\% $\mathrm{CI}=19.98-31.37)$ in $\mathrm{S}+$ vs 15.23 months $(95 \% \mathrm{CI}=11.80$ 18.66) for S- patients.

\section{Outcomes}

The primary outcomes were to identify OS for the whole study population, and for each arm of the study (S+ and S-). Analysis was done using the Kaplan-Meier survival curves and compared using Log-rank test.

The secondary outcomes were to compare the clinico-demographical data between Statins +ve vs. Statins -ve among the whole cohort and to compare survival in different gender subgroups (females and males) among S+ vs. S- cohorts.

\section{Statistical analysis}

Categorical variables were reported as absolute numbers (frequency percentages) and compared using Pearson's Chi-square $\left(\chi^{2}\right)$ test. Continuous variables were presented as a median and IQR and compared using Mann-Whitney U test. Cox regression was used to identify factors predicting DFS in both uni- and multivariate analyses. Kaplan-Meier survival curves with $\mathrm{P}$ value $<0.05$ was considered statistically significant. Unadjusted and adjusted Cox proportional hazard ratios (HR) and 95\% confidence intervals (95\% CI) were conducted as confirmatory tests to the results obtained in K-M curves. Adjustment were done based on different variables combinations (age, gender, race and stage) with statins therapy. Statistical analyses were conducted using "survival" package in R (version 3.3.3 R Project for Statistical Computing). 
Table 1. Showing the Demographics of the Patients

\begin{tabular}{|c|c|c|c|c|c|}
\hline Variables & Groups & $\mathrm{S}-(\mathrm{n}=159)$ & $\mathrm{S}+(\mathrm{n}=41)$ & Total $(n=200)$ & P-value \\
\hline Age & Continuous variable & $61(55-68)$ & $64(61-74)$ & $61(55-69)$ & 0.0003 \\
\hline \multicolumn{6}{|l|}{ Median (IQR) } \\
\hline \multirow[t]{2}{*}{ Gender n (\%) } & Females & $71(44.7)$ & $19(46.3)$ & $90(45.0)$ & 0.846 \\
\hline & Males & $88(55.3)$ & $22(53.7)$ & $110(55.0)$ & \\
\hline \multirow[t]{2}{*}{ Race n (\%) } & White/Caucasian & $69(71.1)$ & $25(71.4)$ & $94(71.2)$ & 0.974 \\
\hline & Non-white & $28(28.9)$ & $10(28.6)$ & $38(28.8)$ & \\
\hline \multirow[t]{3}{*}{ Histology n (\%) } & Adenocarcinoma & $83(56.1)$ & $20(48.8)$ & $103(55.4)$ & 0.517 \\
\hline & SCC & $43(29.7)$ & $16(39.0)$ & $59(31.7)$ & \\
\hline & Others & $19(13.1)$ & $5(12.2 \%)$ & $24(12.9)$ & \\
\hline \multirow[t]{2}{*}{ Stage n $(\%)$} & Stage I-III & $58(38.9 \%)$ & $23(62.2 \%)$ & $81(43.5)$ & \\
\hline & Stage IV & $91(61.1 \%)$ & $14(37.8 \%)$ & $105(56.5)$ & 0.011 \\
\hline Median OS & In months & $13.7(9.13-37.7)$ & $15.6(9.7-N A)$ & $14.9(10.1-26.9)$ & 0.652 \\
\hline Median OS (Females) & In months & $9.67(7.35-11.98)$ & NR & ------- & 0.027 \\
\hline Median OS (Males) & In months & $26.90(9.57-44.23)$ & $9.70(2.90-28.22)$ & --------- & 0.155 \\
\hline
\end{tabular}

\section{Results}

Clinico-demographical data

A total of 200 patients were recruited in this analysis, $\mathrm{S}+$ group $=41$ patients. Females represented $45 \%$ of the population, and $71.2 \%$ were White/Caucasian. Median age of the study population was 64 years (IQR; 55-69). Adenocarcinoma predominated the histological types at $55.4 \%$, followed by for squamous cell carcinoma at $31.7 \%$ and $56.5 \%$ of the patients were stage IV. (see Table 1).

Compared to $\mathrm{S}+, \mathrm{S}-$ patients were more likely to be younger 61years (IQR; 55-68; $\mathrm{P}<0.001$ ). Stage IV

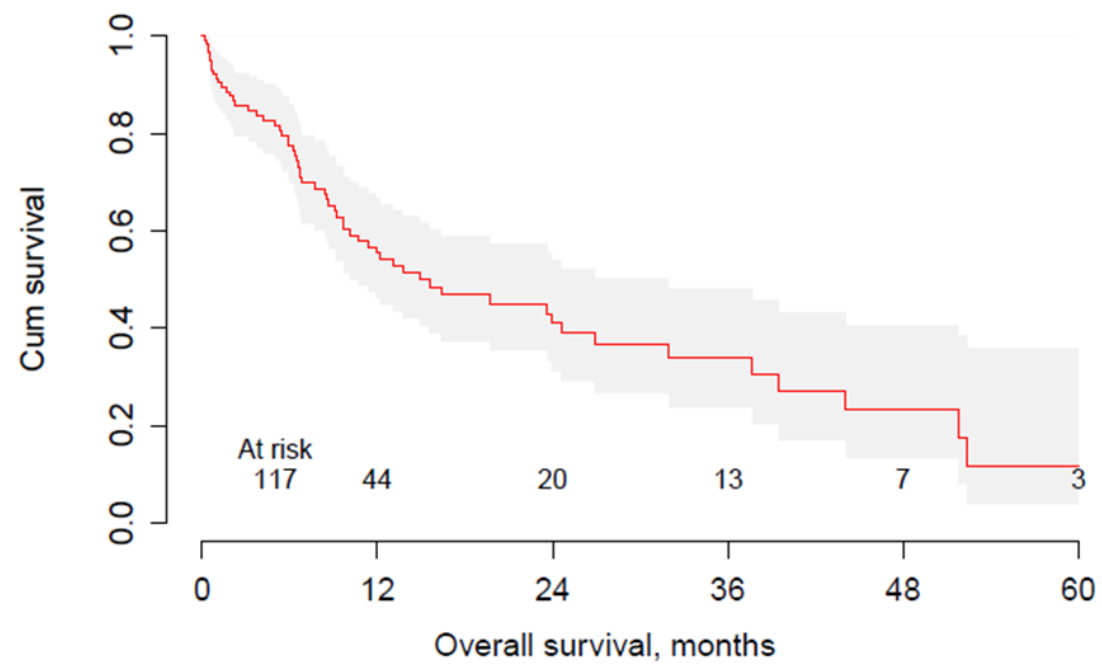

Figure 1. Showing Survival Curves of the Whole Study Population

Table 2. Showing Statin Variable Adjusted Statin Effect on OS

\begin{tabular}{lcccc}
\hline Variable & P-value & HR & Lower 95\%CI & Upper 95\%CI \\
\hline Statin effect & 0.723 & 0.906 & 0.523 & 1.568 \\
Statin effect adjusted to age & 0.606 & 0.86 & 0.485 & 1.526 \\
Statin effect adjusted to gender & 0.702 & 0.898 & 0.517 & 1.56 \\
Statin effect adjusted to race & 0.893 & 0.956 & 0.497 & 1.84 \\
Statin effect adjusted to stage & 0.863 & 1.052 & 0.592 & 1.869 \\
Statin effect adjusted to age \& race & 0.872 & 0.945 & 0.473 & 1.888 \\
Statin effect adjusted to age \& gender & 0.57 & 0.845 & 0.473 & 1.509 \\
Statin effect adjusted to gender \& race & 0.923 & 0.968 & 0.501 & 1.87 \\
Statin effect adjusted to age, gender \& race & 0.907 & 0.959 & 0.477 & 1.93 \\
Statin effect adjusted to age, gender, race \& stage & 0.778 & 1.109 & 0.542 & 2.267 \\
\hline
\end{tabular}




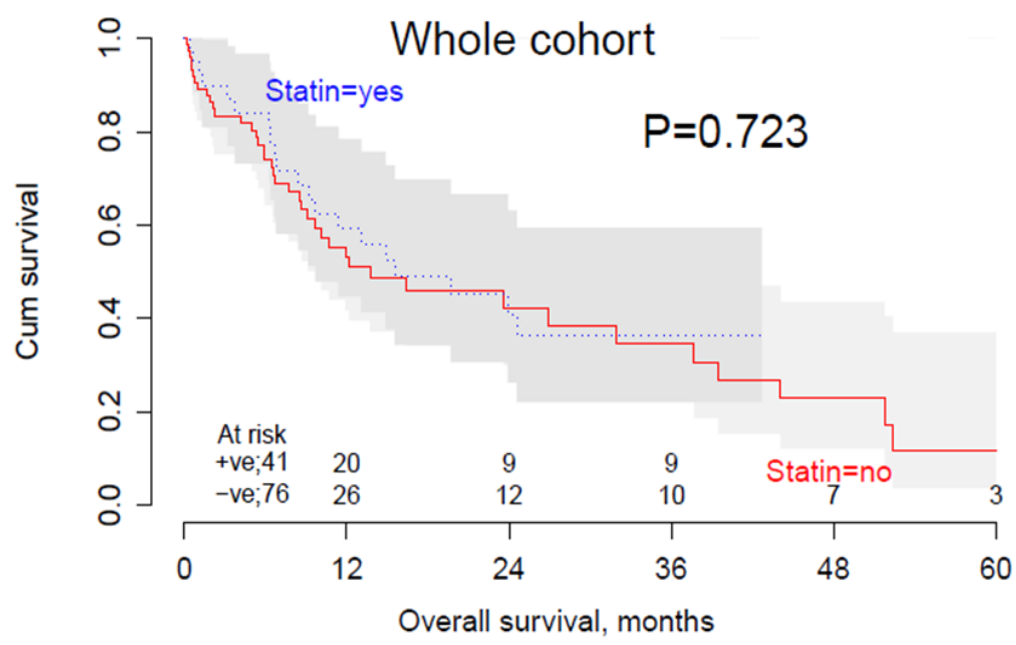

Figure 2. Showing the OS Difference between Both Arms of the Study

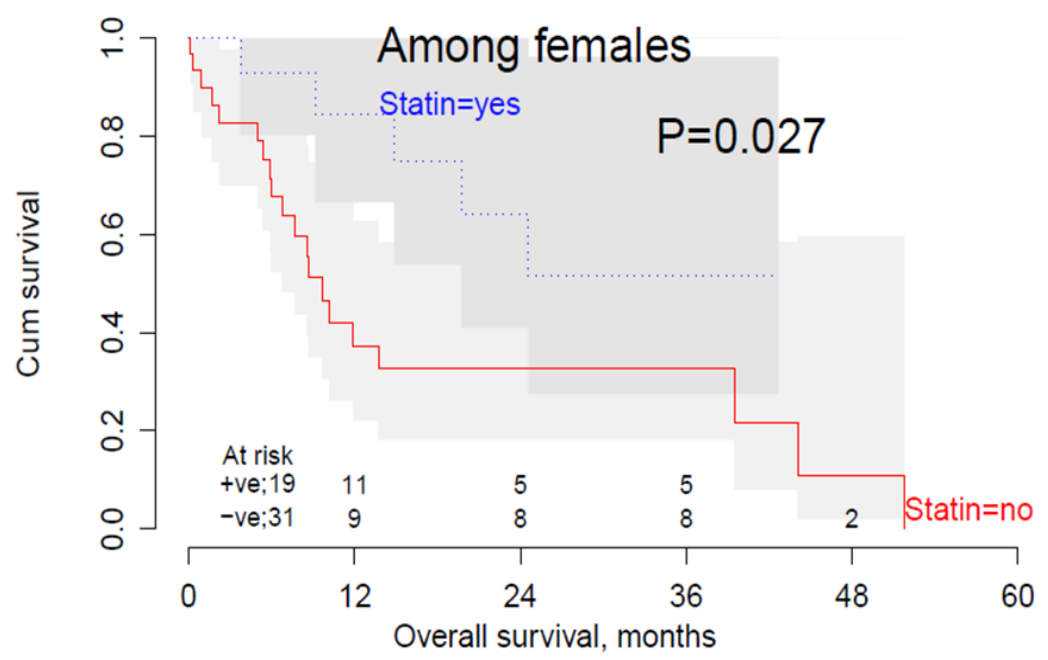

Figure 3. Showing Survival Advantage in OS for Females who Receive Statin

predominated in $\mathrm{S}-$ group $(61.1 \%$ vs $37.8 \% ; \mathrm{P}=0.011)$. However, there was no difference regarding gender, race or histology.

\section{Survival}

The median OS for the whole study population was 14.9 months (95\%CI 10.1-26.9)-see Figure 1.

The median OS was lower in S- vs S+ was 13.7 vs 15.6 months, respectively $(\mathrm{P}=0.652)$. In Figure 2 : unadjusted Cox proportional hazard ratio was $0.91(0.52-1.57)$.

Different subgroup analyses were conducted and revealed better survival for females on statins ( $\mathrm{S}+$ vs $\mathrm{S}-$ is NR vs 9.67, 95\%CI 7.35-11.98, $\mathrm{P}=0.027$ )-see Figure 3. However, there were overlapping confidence intervals in the K-M curves that undermine the strength of this result. As a confirmatory step, we did Cox proportional hazard ratio adjusted to gender that revealed absence of significance in $\mathrm{S}+(\mathrm{HR}=0.90,95 \% \mathrm{CI}=52-1.56)$-see Table 2.

After different types of adjustments, $\mathrm{S}+$ was not associated with any survival advantages $(\mathrm{P}>0.05)$.

\section{Discussion}

In this study, we have focused on sub population of lung cancer patients from 2011 to 2017 presented to our institute who received statins during the duration of active cancer treatment. Previously, we published clinical studies that focused on our experience with lung cancer patients with rare and less common presentation such as clear cell lung cancer, pulmonary sarcomatoid and large cell neuroendocrine carcinoma of the lung (Eldessouki et al., 2018; Eldessouki 2018b; Karim et al., 2018). Statins are established FDA approved drugs that have been well studied in terms of safety, dosage, and toxicity. For the past several decades there have been increasing blood cholesterol and lipid levels among Americans, making statins a commonly prescribed and relatively inexpensive drug. There have been numerous studies that show statin use as either a benefit or no harm in cancer treatment (Oudat et al., 2002; Seckl et al., 2017). A study looking at the entire Danish population who had received a diagnosis of cancer from 1995-2007 observed that all-cause mortality among patients with cancer who were taking statins was reduced by $15 \%(95 \% \mathrm{CI}, 13-17)$ and the multivariable-adjusted hazard ratio for death from cancer 
among statin users, as compared with patients who had never used statins, was 0.85 (95\% CI, 0.82-0.87) (Nielsen et al., 2012). Katz et al., (2005) assessed if statins may enhance the efficacy of neodjuvant chemoradiation in rectal cancer by analyzing pathologic complete response rates (no microscopic evidence of tumor). They found that the rates with statin use were $30 \%$ compared to $17 \%$ without $(\mathrm{p}=0.10)$ and the OR for statin use was $4.2(95 \%$ CI, 1.7-12.1; $\mathrm{p}=0.003)$ concluding that the use of statins was associated with an improved pathologic complete response rate. Mace et al., (2013) also found statin therapy to be associated with an improved response of rectal cancer to neoadjuvant chemoradiation by showing that patients in the statin cohort had a lower median regression grade ( 1 vs $2, p=0.01$ ) and were more likely to have a better response (grades $0-1$ vs 2-3) than those not taking a statin (65.7\% vs $48.7 \%, \mathrm{p}=0.004)$.

Fiala et al., (2015) found that statins augment the efficacy of EGFR-TKIs in patients with advanced stage NSCLC with KRAS mutation. The median progression free survival for patients treated with EGFR-TKI without statin was 1 -month vs 2 months for patients treated with combination of EGFR-TKI and statin $(p=0.025)$. The median OS for patients treated with EGFR-TKI without statin was 5.4 months vs 14 months for patients treated with combination of EGFR-TKI and statin but and the difference was not statistically significant $(p=0.130)$.

With laboratory research showing that statins increase the effects of chemotherapy on lung cancer cell growth in vitro, LUNGSTAR, a clinical trial in the UK, looked at chemotherapy with and without pravastatin to treat SCLC. To date, LUNGSTAR is one of the largest randomized trials of statin therapy in cancer patients. The study administered pravastatin or matching placebo $(40 \mathrm{mg})$ orally once per day from the start of standard chemotherapy for 2 years unless disease progression or intolerable toxicity occurred. The 2-year OS rate in pravastatin patients was found to be $13.2 \%$ (95\% CI, 10.0 to 16.7 ) versus $14.1 \%$ (95\% CI, 10.9 to 17.7$)$ in placebo with a hazard ratio of $1.01(95 \% \mathrm{CI}, 0.88$ to $1.16 ; \mathrm{P}=.90)$. The median OS was 10.7 months $v 10.6$ months, respectively and the median progression-free survival was 7.7 months v 7.3 months, respectively. They concluded that when combined with standard platinum chemotherapy pravastatin use did not improve outcomes in patients with SCLC, nor in those with limited stage or extensive stage disease (Seckl et al., 2017). This retrospective study, while showing a possible benefit of statin use with an increased OS of 648 days in patients taking statins vs 539 in the control, did not reach statistical significance ( $\mathrm{p}$ value $=0.513, \mathrm{HR}=0.840$ ).

In conclusion, while showing an increase in overall survival in patients with advanced lung cancer, the results of this study did not reach statistical significance. This could be due for the small sample size of this retrospective study. It is also important to note that neither the specific statin used nor the dosages were reported. As atorvastatin, lovastatin, and simvastatin are lipophilic, there may be differences in their pharmacology when compared to hydrophilic statins. Larger prospective studies are warranted to further investigate the role of statins in lung cancer.
List of abbreviations

CI: confidence interval

EGFR: epidermal growth factor reciptor

FDA: Food and Drug administration

HR: hazard ratio

IQR: interquartile Range

NR: not reached.

OS: overall survival

$\mathrm{S}+$ : statin receiving arm

S-: Control arm

SCLC: small cell lung cancer

TKI: tyrosine kinase inhibitor

\section{Acknowledgments}

\section{Conflicts of interest}

Authors declare no conflict of interest.

\section{Ethics and consent}

During the process of data collection, all patients' data were properly anonymized and informed consent was obtained prior to data collection. The study was approved by the review boards and ethical committee of University of Cincinnati Medical Center and was designed in concordance with declaration of Helsinki.

\section{Funding}

The study was funded by an interior grant.

\section{References}

Abdel Karim N, Eldessouki I, Taftaf A, et al (2018). GNQ209P mutation in metastatic uveal melanoma and treatment outcome. Case Rep Oncol Med, 2018, 1-5.

Abdel Karim NF, Ahmad I, Gaber O, et al (2019). Phase I trial of chloroquine (CQ)/hydroxychloroquine (HCQ) in combination with carboplatin-gemcitabine $(\mathrm{CG})$ in patients with advanced solid tumors. J Clin Oncol, 37, 3027.

Agustsson T, Rydén M, Hoffstedt J, et al (2007). Mechanism of increased lipolysis in cancer cachexia. Cancer Res, 67, 5531-7.

Altorki NK, Kamel MK, Narula N, et al (2016). Anatomical segmentectomy and wedge resections are associated with comparable outcomes for patients with small cT1N0 nonsmall cell lung cancer. $J$ Thorac Oncol, 11, 1984-92.

Anon SEER Cancer Statistics Review 1975-2006 - Previous Version - SEER Cancer Statistics. Available at: https:// seer.cancer.gov/archive/csr/1975_2006/ [Accessed July 24, 2017].

C. for Disease Control (2017). CDC - NCHS - National Center for Health Statistics. Available at: https://www.cdc.gov/nchs/ index.htm [Accessed February 27, 2018].

Cerne D, Prodan Zitnik I, Sok M (2010). Increased fatty acid synthase activity in non-small cell lung cancer tissue is a weaker predictor of shorter patient survival than increased lipoprotein lipase activity. Arch Med Res, 41, 405-9.

Chan KKW, Oza AM, Siu LL (2003). The statins as anticancer agents the statins as anticancer agents. Maedica (Buchar), 9, 10-19.

Eldessouki I, Gaber O, Namad T, et al (2018). Small or non-small cell lung cancer based therapy for treatment of large cell neuroendocrine cancer of the lung? University of Cincinnati Experience. J Oncol, 2018, 1-6.

Eldessouki I, Gaber O, Riaz Muhammad K, Wang Jiang AKN 
(2018a). Clinical presentation and treatment options for clear cell lung cancer: University of Cincinnati a case series and literature review of clear cell lung cancer. https://doi. org/10.22034/APJCP.2018.19.9.2373.

Eldessouki I, Gaber O, Riaz MK, Wang Jiang AKN (2018b). Clinical presentation and treatment options for clear cell lung cancer: University of Cincinnati A case series and literature review of clear cell lung cancer. Asian Pac J Cancer Prev, 19, 2373-6.

ENDO A (2010). A historical perspective on the discovery of statins. Proc Japan Acad Ser B, 86, 484-93.

Fiala O, Pesek M, Finek J, et al (2015). Statins augment efficacy of EGFR-TKIs in patients with advanced-stage non-small cell lung cancer harbouring KRAS mutation. Tumor Biol, 36, 5801-5.

Gaber O, Eldesouki I, Magdy M, et al (2019). Targeted therapy for KRAS mutant non-small cell lung cancer. Available at: www.ClinicalTrials.gov [Accessed July 4, 2019].

Gaber O, Eldessouki I, Magdy M, et al (2018). Virtosome: Is it the answer to liquid biopsy dilemma?. Correspondence to. Available at: https://www.cientperiodique.com/article/ CPQCR-1-3-18.pdf [Accessed November 8, 2018].

Galetti M, Petronini PG, Fumarola C, et al (2015). Effect of ABCG2/BCRP expression on efflux and uptake of Gefitinib in NSCLC cell lines W. J. Huss (ed.). PLoS One, 10, e0141795.

Graaf MR, Richel DJ, van Noorden CJF, et al (2004). Effects of statins and farnesyltransferase inhibitors on the development and progression of cancer. Cancer Treat Rev, 30, 609-41.

Hajar R (1995). Statins: Past and present history of medicine. Heart Views, 12, 121-7.

Hanai JI, Doro N, Sasaki AT, et al (2012). Inhibition of lung cancer growth: ATP citrate lyase knockdown and statin treatment leads to dual blockade of mitogen-activated protein Kinase (MAPK) and Phosphatidylinositol-3-kinase (PI3K)/ AKT pathways. J Cell Physiol, 227, 1709-20.

Hassan R, Gulati S, Mahender Y, et al (2017). Impact of low molecular weight Heparin on overall survival in patients with advanced lung cancer: A retrospective study. Am J Clin Exp Med, 5, 173.

Hoque A, Chen H, Xu X-C (2008). Statin induces apoptosis and cell growth arrest in prostate cancer cells. Cancer Epidemiol Biomarkers Prev, 17, 88-94.

Jemal A, Siegel R, Xu J, et al (2010). Cancer statistics, 2010. CA Cancer J Clin, 60, 277-300.

Junior LS (2015). Development of antidepressants as novel agents to treat small cell lung cancer development of antidepressants as novel agents to treat small cell lung cancer approved for public release; distribution unlimited. Available at: http://www.dtic.mil/dtic/tr/fulltext/u2/1016343. pdf [Accessed February 27, 2018].

Karim NA, Schuster J, Eldessouki I, et al (2018). Pulmonary sarcomatoid carcinoma: University of Cincinnati experience. Oncotarget, 9, https://doi.org/10.18632/oncotarget.23468.

Karim N, Eldessouki I, Yellu M, et al (2017). A case study in advanced lung cancer patients with vimentin over expression. Clin Lab, 63, https://doi.org/10.7754/CIin. Lab.2017.170201.

Katz MS, Minsky BD, Saltz LB, et al (2005). Association of statin use with a pathologic complete response to neoadjuvant chemoradiation for rectal cancer. Int $J$ Radiat Oncol, 62, 1363-70.

Khanzada UK, Pardo OE, Meier C, et al (2006). Potent inhibition of small-cell lung cancer cell growth by simvastatin reveals selective functions of Ras isoforms in growth factor signalling. Oncogene, 25, 877-87.

Mace AG, Gantt GA, Skacel M, et al (2013). Statin therapy is associated with improved pathologic response to neoadjuvant chemoradiation in rectal cancer. Dis Colon Rectum, 56, 1217-27.

Merino Salvador M, Gómez de Cedrón M, Merino Rubio J, et al (2017). Lipid metabolism and lung cancer. Crit Rev Oncol Hematol, 112, 31-40.

N K, E-M B, O G, et al (2017). A phase I study of the non-receptor tyrsine kinase inhibitor (NKI) Bosutinib in Combination with Pemetrexed in Patients with Advanced Solid Tumors. J Thorac Oncol, 12, S904.

Nielsen SF, Nordestgaard BG, Bojesen SE (2012). Statin use and reduced cancer-related mortality. $N$ Engl J Med, 367, 1792-1802.

Noto A, Raffa S, De Vitis C, et al (2013). Stearoyl-CoA desaturase-1 is a key factor for lung cancer-initiating cells. Cell Death Dis, 4, e947.

Osugi J, Yamaura T, Muto S, et al (2015). Prognostic impact of the combination of glucose transporter 1 and ATP citrate lyase in node-negative patients with non-small lung cancer. Lung Cancer, 88, 310-8.

Oudat R, Keating MJ, Lerner S, et al (2002). Significance of the levels of bone marrow lymphoid infiltrate in chronic lymphoc leukemia patients with nodular partial remission. Leukemia, 16, 632-5.

Page B, Page M, Noel C (1993). International journal of oncology. University of Crete, Faculty of Medicine, Laboratory of Clinical Virology. Available at: https://www. spandidos-publications.com/ijo/23/4/1055 [Accessed February 27, 2018].

Rahouma M, Kamel M, Abouarab A, et al (2018). Lung cancer patients have the highest malignancy-associated suicide rate in USA: A population-based analysis. Ecancermedicalscience, 12, https://doi.org/10.3332/ ecancer.2018.859.

Rahouma M, Kamel M, Narula N, et al (2017). Pulmonary sarcomatoid carcinoma: an analysis of a rare cancer from the Surveillance, Epidemiology, and End Results database. Eur J Cardio-Thoracic Surg, https://doi.org/10.1093/ejcts/ezx417. Seckl MJ, Ottensmeier CH, Cullen M, et al (2017). Multicenter, phase III, randomized, double-blind, placebo-controlled trial of pravastatin added to first-line standard chemotherapy in small-cell lung cancer (LUNGSTAR). J Clin Oncol, 35, 1506-14.

Visca P, Sebastiani V, Botti C, et al (2004). Fatty acid synthase (FAS) is a marker of increased risk of recurrence in lung carcinoma. Anticancer Res, 24, 4169-73.

Yoh K, Ishii G, Yokose T, et al (2004). Breast cancer resistance protein impacts clinical outcome in Platinum-Based chemotherapy for advanced non-small cell lung cancer. Clin Cancer Res, 10, 1691-7.

This work is licensed under a Creative Commons AttributionNon Commercial 4.0 International License. 